\title{
Efficacy of Anti-Thrombogenic Coating in Decreasing the Rate of Clot Formation on Indwelling Intravascular Devices during Intra-Cardiac Ablation
}

\author{
Omar Yasin ${ }^{1}$, Alan Sugrue ${ }^{1}$, Koji Kadowaki ${ }^{2}$, Hajime Sakakibara², Takahiro Yagi², So Kakiyama², Kazuhiro \\ Tanahashi ${ }^{2}$, Dorothy J Ladewig ${ }^{1}$, Samuel J Asirvatham ${ }^{1}$ and Suraj Kapa ${ }^{1 *}$
}

${ }^{1}$ Department of Cardiovascular Medicine, Mayo Clinic College of Medicine, USA

${ }^{2}$ Toray Industries, Inc., 1-1, Nihonbashi-Muromachi 2-chome, Chuo-ku, Japan

*Corresponding author: Suraj Kapa, Department of Cardiovascular Medicine,

Mayo Clinic College of Medicine, Rochester, MN, USA.

Received Date: August 18, 2021

Published Date: September 13, 2021

\begin{abstract}
Background: Cardiac catheter ablation is associated with risk of thromboembolism such as stroke. Potential sources of embolism include thrombosis on guidewires, ablation catheters, and sheaths. In this study, we developed and tested a novel anti-thrombogenic coating to reduce the rate of thrombosis on indwelling intravascular devices.

Methods: A prototype cerebral protection device (CPD) was placed in the ascending aorta for 90 minutes in a swine animal model. Extent of clot formation/adhesion on the CPD was quantified using a planar light source and a high-resolution camera to measure clot adhesion ratio after device extraction. Clot adhesion ratios are compared between CPDs that were coated with our novel anti-thrombogenic coating and placebo/non-coated devices.

Results: We successfully developed CPD devices with anti-thrombogenic coating and deployed them in the ascending aorta for 90 minutes in seven animal experiments. There were no hemodynamic or radiologic evidence of obstruction to blood flow in any device. Clot adhesion ratio was $6.7 \pm 4.61 \%$ on coated devices compared to $19.3 \pm 15.77 \%$ on non-coated devices ( $65 \%$ reduction in clot adhesion ratio on coated CPDs).

Conclusions: Our novel anti-thermogenic coating is associated with less clot adhesion on a prototype CPD placed in the ascending aorta for a 90 minute procedure in a swine animal model.
\end{abstract}

Keywords: Embolism; Catheter ablation; Cerebro-protective devices; Cardiac embolism

\section{Introduction}

Thromboembolism poses a serious risk to patients undergoing catheter ablation [1]. In addition to risk of overt stroke and myocardial infarction, what were once thought to be "asymptomatic" brain lesions detected on magnetic resonance imaging (MRI) are now shown to be associated with long term risk of dementia and cognitive decline [2-4]. In fact, these brain lesions are quite common and, depending on ablation modality used and MRI definition, they can be present in up to a third of patients undergoing pulmonary vein isolation for atrial fibrillation [2-6]. Sources of embolism during cardiac ablation include thrombosis that occurs on guidewires, ablation catheters, and sheaths. Thrombosis can also result from direct heating or cooling of the blood pool which directly denatures proteins (including fibrin). Thrombosis can also result from disruption of the epithelial layer at the ablated site [7,8]. Non thrombogenic sources of emboli include char, necrotic tissue, and foreign material $[1,9]$. Air can also embolize as it can be introduced 
during catheter exchange, irrigation, suctioning, flushing and medication administration [1,10]. Anticoagulation and careful sheath and catheter manipulation can reduce the risk of formation of embolic material during catheter ablation. Nonetheless, innovative approaches to reduce the risk of formation of such material during cardiac ablation include using non-thermal energy sources for ablation and applying continuous negative charge on catheters to repel the negatively a charged fibrin molecules [11-14]. However, once formed, cerebral protection devices (CPD) have proven their efficacy in capturing and containing thrombus and other emboli from the systemic circulation during intervascular interventions [9,15-20]. Despite proving their efficacy in capturing embolic material, the use of these devices might be limited by dwell time due to the risk device clot occlusion or decreased blood flow [21]. To further improve safety of intravascular procedures, we propose an innovative approach to reduce the thrombogenic properties of any intravascular device including CPDs. In this study, our team developed and tested a novel anti-thrombogenic coating that can be applied to medical devices to reduce the rate of thrombosis on their surface. We hypothesize that the application of our antithrombogenic agent can decrease clot adhesion on indwelling intravascular devices. To test this hypothesis, we designed a prospective feasibility study in a swine animal model to compare the clot adhesion ratio on coated versus uncoated prototype CPD device placed in the ascending aorta.

\section{Material and Methods}

The study design had two parts each containing multiple phases. In Phase I, we fabricated the CPD devices and assessed the capture efficiency ex-vivo in a circuit containing saline. In Phase II, we conducted all the in-vivo assessments of device patency and efficiency. Phase II experiments were conducted in Japan and USA as outlined below.

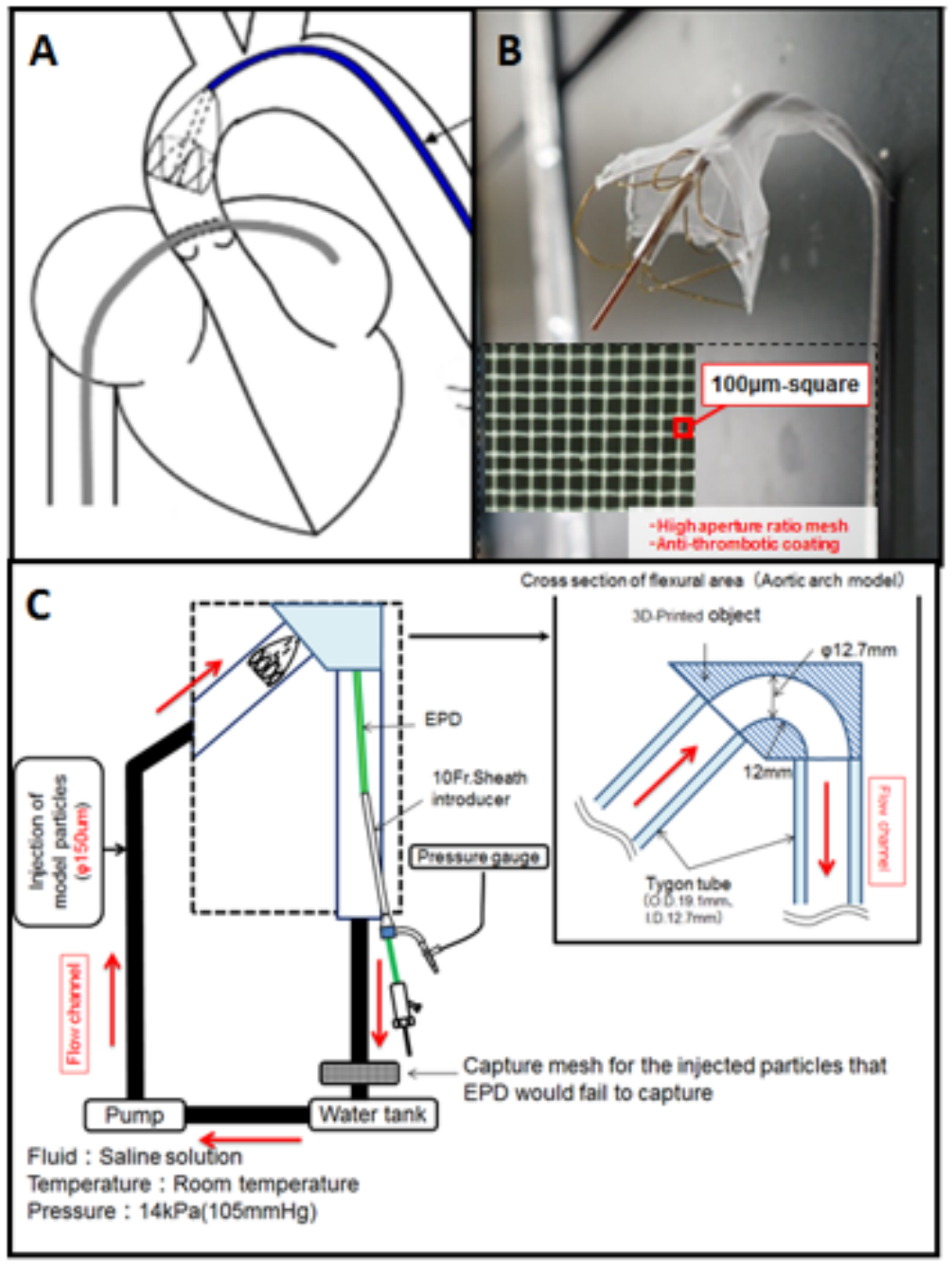

Figure 1: Cerebral protection device. A- Cartoon representation demonstrating the approximate device position and orientation in the ascending aorta. B- Our prototype cerebral protection device (CPD) C- A-Close loop circuit for Ex-Vivo quantification of capture efficiency test. 


\section{Phase I}

Device fabrication \& coating: To gain insight into the efficacy of the anti-thrombogenic coating, we wanted a large enough surface area that would allow for quantification of the clot burden on a representative device. Given the relatively small size of catheter tips commonly used for ablation and the instability of clot adhesion at those surfaces from constant manipulation and/or irrigation, we elected to use a prototype CPD placed in the ascending aorta. The novel CPD consists of a nickel-titanium frame with a polyester mesh (Figure 1). To prevent early occlusion, a novel antithrombogenic polymer coating that inhibits both enzymatic activity of thrombin and platelet adhesion was applied to the mesh (Toray Industries, Inc., Tokyo). Each pore in the polyester mesh was $100 \mu \mathrm{m}$ in length on one side. The polyester yarn is $27 \mu \mathrm{m}$ in diameter. The mesh filter is mounted on a flower- shaped nickel-titanium frame to allow the filter to conform to the interior diameter of each ascending aorta. The diameter of the frame ring is 18 to $20 \mathrm{~mm}$. The points of curvature of frame ring are connected to a core shaft with four polyester threads (distal part) and four stainless steel threads (proximal part). An outer tube, which consists of a polyimide and polyether block amide 6 French outer diameter tube is set over the core shaft. The filter ring can close when the operator pulls the core shaft along the outer tube. The filter is delivered to the ascending aorta via the femoral artery using a 0.014-inch guidewire. Two different types of mesh were used (1) non-coated, and (2) coated devices with the aforementioned anti-thrombogenic coating Briefly, heparin was loaded to the surface of the polyester mesh of the CPD through ionic interaction. The surface of the polyester mesh was covalently modified with polyamine by soaking it in polyamine aqueous solution (5.0 wt\%). After rinsing off the excess polyamine, the modified polyester mesh was incubated in heparin aqueous solution ( $0.75 \mathrm{wt} \%$ ). The surface amount of heparin was quantified with anti-factor Xa activity, using Testzym® Heparin S (Sekisui Medical Co., Ltd., Tokyo, Japan).

Capture efficiency testing: A closed loop circuit was created which allowed for placement of our CPD device through which saline flowed. Small $(0.25 \mathrm{X} 0.25 \mathrm{~mm})$ radiopaque particles were injected into the circuit to demonstrate their capture by the CPD device. Capture efficacy was quantified by measuring the amount of particles that were not captured by the CPD and were instead captured on a mesh distal to the device that was also part of the circuit.

\section{Phase II}

Our primary outcomes for the in-vivo experiments were to compare coated and uncoated CPD patency throughout a 90-minute procedure and to quantify the amount clot formation/adhesion on the CPD. Our secondary outcomes were to assess the efficacy of the CPD in capturing emboli generated proximal to the device during radiofrequency (RF) ablation and particulate injection into the left ventricular outflow tract. The secondary outcomes were not assessed in every experiment. A total of eight animal experiments were conducted (Phase II). The first three experiments were conducted in Japan at the Fukushima Medical Device Development Support Centre on swine animal model, Zenno Premium Pig (ZPP) that was made by crossing Large Yorkshire and Land breed. All experiments were conducted in accordance with the Biological Evaluation of Medical devices -Part2: Animal welfare requirements. The last five experiments were conducted at Mayo Clinic (Rochester, MN, US) also using female swine model (Sus scrofa). All procedures at Mayo Clinic were reviewed and approved by the Institutional Animal Care and Use Committee and experiments were conducted according to laboratory animal use and care regulations. One animal study was excluded due to early animal demise secondary to myocardial infarction from an air embolus through the trans-septal sheath very early in the experiment (immediately after trans-septal access was established). Animals were fasted for 12 hours prior to surgery and maintained under general anesthesia using isoflurane (1-3\%) after induction with midazolam, medetomidine and thiamylal sodium at the Fukushima Medical Device Development Support Centre. Propofol and telazole were used for anesthesia induction at Mayo Clinic. Femoral venous and arterial accesses were established for CPD placement and ablation catheters. Internal jugular access was used for intra-cardiac echocardiogram catheters. Heparin was used to maintain an activated clotting time (ACT) of greater than $300 \mathrm{sec}$ prior to the procedure.

Device patency: Once the ACT was greater than $300 \mathrm{sec}$, the femoral arterial access was used to advance a coated or un-coated CPD into the arterial circulation. Under direct fluoroscopic and intracardiac echocardiogram guidance, the CPD was advanced and deployed in the ascending aorta just above the sinus of Valsalva (Figure 2). Once complete, venous access was obtained and an Agilis sheath was used to establish trans-septal access into the left atrium. The CPDs were allowed to indwell for 90 minutes in the aorta to assess a baseline clot adhesion ratio on the CPD. Contrast injection into the left atrium were performed every 30 minutes to confirm CPD patency. After the 90 minutes were complete, the CPD device was removed and replaced with a new device that was placed in the same location. The first three experiments conducted in Japan, during each experiment one coated and one uncoated CPD were allowed to indwell for at least 90 minutes in each animal. This experiment was replicated once at Mayo Clinic. Thus, four pairs of CPDs (one coated and one uncoated) were tested in four animals without any ablations.

Capture efficiency with radiofrequency ablation: In four animal experiments RF ablation was performed in the left atrium while a CPD was placed in the aorta. For ablation, a standard non-irrigated ablation catheter (BlazerTM II, Boston Scientific Corporation) or open irrigated ablation catheter (THERMOCOOL®, 
Biosense Webster) was advanced into the left atrium through the trans-septal access (Figure 3). Five RF ablations were performed at $50 \mathrm{~W}$ for 30 seconds. After five ablations were complete in the left atrium, the CPD device was allowed to indwell at the same location to complete at least 90 minutes. Each animal had two sets of ablations preformed, using two different CPDs.

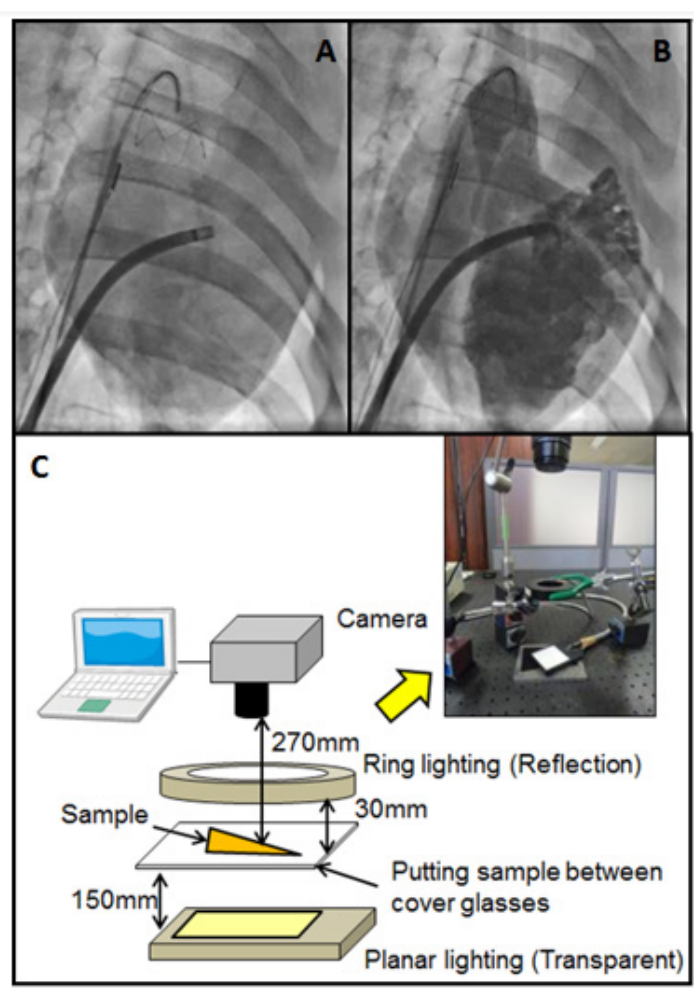

Figure 2: Device position and clot adhesion ratio. A-Anterior-posterior fluoroscopy view of the CPD in the aorta. B- Contrast injection through CPD device demonstrating adequate position and patency. C- Clot adhesion ratio quantification apparatus.



Figure 3: Capture efficiency. A- Cartoon representation of the cerebral protection device (CPD) device in the ascending aorta and a multipurpose catheter for In-Vivo capture efficiency test. B- Anterior posterior fluoroscopic view of the multipurpose catheter injecting particulate matter in the left ventricular outflow tract with CPD in place. C-Anterior-posterior view of the CPD device in place during left atrial ablation through transseptal access. D\&E- Small thrombi collected after rinsing two of CPD devices that were used during non-irrigated radiofrequency ablation in the left atrium. F\&G- Small thrombi collected after rinsing two of CPD devices that were used during irrigated radiofrequency ablation in the left atrium. 
Capture efficiency with particulate injection: Once the ablations were complete and CPD was removed, a last device was placed in the aorta for the capture efficiency test. This test was performed at the end of three of the animal experiments. During this portion, a multipurpose catheter was advanced through the trans-septal access into the left ventricular outflow tract (LVOT) just under the aortic valve. A predetermined number of small $[0.25$ $\mathrm{X} 0.25 \mathrm{~mm}$ ) radiopaque particles were injected in the LVOT. The device was then removed, and the amount of radiopaque material captured by the filter was quantified.

\section{Outcome Assessment}

Device patency (Phase IIA) was assessed by injecting contrast proximal to the device at predetermined intervals (described above). Once the CPD was removed, each was rinsed with $0.9 \%$ saline to dislodge any non-adherent clot that was collected using a strainer. The burden of non-adherent clot served as a surrogate for emboli from ablation that was captured by the CPD and was qualitatively evaluated. After rinsing with saline, the device itself was stored in $10 \%$ formalin solution. The mesh filter was removed from the nickel-titanium frame of each catheter for analysis. Each CPD sample was analyzed using a planar light source and a highresolution camera, the surface area covered by clot was calculated relative to the total surface area of the CPD filter to calculate the clot adhesion ratio by the following equation. Clot adhesion ratio
$(\%)=$ (the area of clot adhesion/ the overall area of the filter mesh) x 100 All data was reported as mean ratio \pm standard deviation. No statistical analysis was performed given the low sample size.

\section{Result}

\section{Capture efficiency}

Three experiments were conducted assessing the capture efficiency of the CPT device ex-vivo. The capture efficiency was calculated to be $95.17 \pm 0.67 \%$. The mean number of particles that were not captured was $3.5 \pm 1.56 \%$. In addition, $1.2 \pm 1.36 \%$ particles were lost at the time of CPD removal.

\section{Device patency}

A total of six CPDs were included in the analysis for clot adhesion ratio. Three animals were in Japan and one at Mayo Clinic. The device was successfully deployed in all animals with no device related acute complications. Contrast injections at time of device placement, 30 minutes, 60 minutes and 90 minutes after device placement have consistently demonstrated good contrast flow through all devices with no evidence of obstruction. The clot adhesion ratio was lower in the anti-thrombogenic coated CPDs compared to uncoated (Figure 4). There was a 65\% reduction in clot adhesion ratio on the uncoated mesh than the coated (clot adhesion ratio of $19.3 \pm 15.77 \%$ vs. $6.7 \pm 4.61 \%$ ).

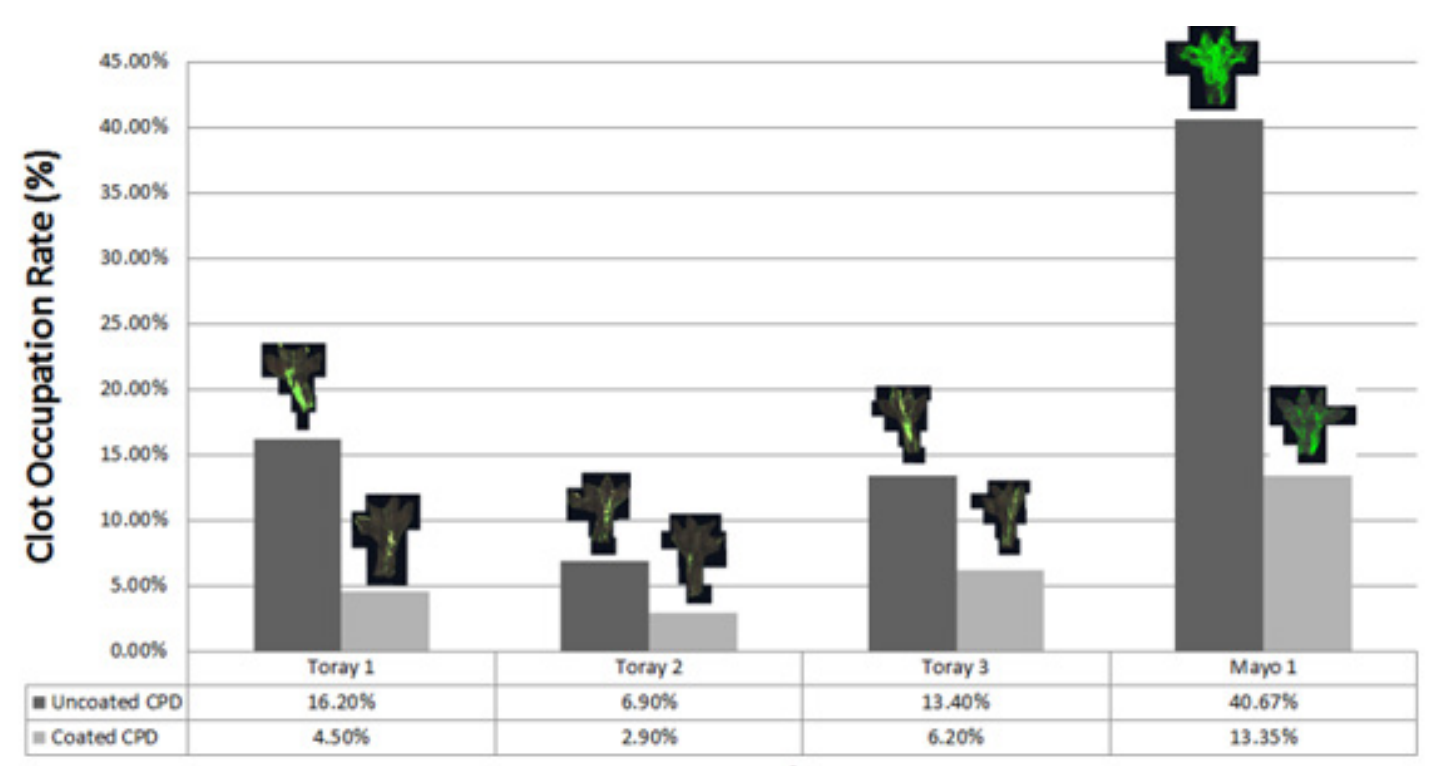

Animal

Figure 4: Clot adhesion ratio. Data for the four animal experiments comparing the clot adhesion rate between cerebral protection devices (CPD) coated with anti-thrombogenic coating versus uncoated. All devices were placed for at least 90 minutes in the sinus of Valsalva. Clot adhesion rate $(\%)=$ (the area of clot adhesion/the overall area of the filter mesh) $\times 100$. Above each bar is the actual image of the CPD device showing clot adhesion. 


\section{Capture efficiency with radiofrequency ablation}

Three coated CPDs were placed for 90 minutes in three experiments without ablation to serve as control. The mean clot

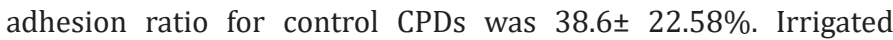
ablation was performed in two experiments (coated CPDs) and clot adhesion ratio was $32.1 \pm 17.52 \%$. Non-irrigated ablation was performed in three animal experiments and a total of four CPDs were used (three coated and one non-coated) clot adhesion ratio was $27.35 \pm 11.60 \%$ with non-irrigated ablation. In summary, mean clot adhesion ratio did not significantly correlate with ablation regardless of whether it was irrigated or non-irrigated ablation. Qualitatively, there was a clear difference in the rate of emboli/ debris within the devices that had ablation versus no ablation. Ablation with irrigation seemed to have less emboli/debris within the devices.

\section{Capture efficiency with particulate injections}

Injection of the radiopaque particles was performed at the end of three animal experiments. Capture efficiency was 43\%, 67.4\% and $94.4 \%$ in the first, second and third experiments. Of note, a good number (not quantified) of the radiopaque material injected in the left ventricle had remained trapped in the left ventricular trabeculations and did not necessarily embolize to the systemic circulation.

\section{Discussion}

In this experiment, we were able to successfully create a novel prototype cerebral protection device with anti-thrombogenic coating and deploy it in the aortic arch for 90 minutes without causing any hemodynamic or radiologic evidence of obstruction to blood flow. The anti-thrombogenic coating was associated with lower clot adhesion ratio on the CPD device compared to noncoated devices. However, no statistical analysis was feasible given the limited number of observations. As for the secondary endpoint, the device was able to maintain its position and capture emboli (from ablation and radiopaque particles) while remaining patent. The variation of clot adhesion ratio of the coated mesh at baseline, made it difficult to distinguish non-ablation related clot versus emboli as a result of ablation (Figure 5). While the capture efficiency is far from perfect, it still remains a significant advantage to the current standard of care-which is no protection at all. Qualitatively, we observed an expected trend towards lower number and size of embolic debris when using irrigated ablation.



Figure 5: Clot adhesion ratio per animal and device. A- Scatter plot summarizing the clot adhesion ratio of all CPD devices stratified by animal with, device and ablation type. B- Scatter plot demonstrating clot adhesion ratio stratified by device and type of irrigation regardless of animal. All devices were placed for at least 90 minutes in the sinus of Valsalva. Clot adhesion ratio $(\%)=($ the area of clot adhesion/ the overall area of the filter mesh) $\times 100$ 
Our experimental design has several limitations. First, being a feasibility study, the low number of data points prevented us from performing any statistical analysis. Second, the device used was a prototype device and despite the ex-vivo testing (which allowed to device to fit almost perfectly in the circuit); its efficacy in capturing debris depends on the individual anatomy of each animal's aorta. Third, it is difficult to ascertain the difference between clots formed on the device versus clot that was captured during ablations. Nonetheless, these initial results create a good foundation to power future experiments to assess the efficacy of the anti-thermogenic coating and to assess the efficiency of future iterations of this CPD In future experiments we aim to assess the efficacy of the antithrombogenic coating on chronically indwelling devices such as left atrial appendage occlusion devices or prosthetic valves. This will not only demonstrate the utility of such coating in low flow situations, such as the left atrium, but also provide insight into the utility of using such coating chronically.

\section{Conclusion}

Our novel anti-thermogenic coating is associated with less clot adhesion on a prototype CPD placed in the ascending aorta for a 90-minute procedure in a swine animal model.

\section{Acknowledgement}

None.

\section{Conflict of Interest}

No conflict of interest.

\section{References}

1. Raviele A, Natale A, Calkins H, John A Camm, Riccardo Cappato, et al. (2012) Venice Chart international consensus document on atrial fibrillation ablation: 2011 update. J Cardiovasc Electrophysiol 23: 890923.

2. Lin Y Chen, Faye L Lopez, Rebecca F Gottesman, Rachel R Huxley, Sunil K Agarwal, et al. (2014) Atrial fibrillation and cognitive decline-the role of subclinical cerebral infarcts: the atherosclerosis risk in community's study. Stroke 45: 2568-2574

3. Kok LC, Mangrum JM, Haines DE, Mounsey JP (2002) Cerebrovascular complication associated with pulmonary vein ablation. J Cardiovasc Electrophysiol 13: 764-767.

4. Ponamgi S, Yasin O, Padmanabhan D, DeSimone C, Asirvatham S (2016) Innovative Approaches to Assess the Impact and Steps to Decrease the Neurological Consequences of Ablation. Journal of Innovations in Cardiac Rhythm Management 7: 2349-2361.

5. Deneke T, Jais P, Scaglione M, Rainer Schmitt, Luigi DI Biase, et al. (2015) Silent cerebral events/lesions related to atrial fibrillation ablation: a clinical review. J Cardiovasc Electrophysiol 26: 455-463.

6. Claudia Herrera Siklódy, Thomas Deneke, Mélèze Hocini, Heiko Lehrmann, Dong-In Shin, et al. (2011) Incidence of asymptomatic intracranial embolic events after pulmonary vein isolation: comparison of different atrial fibrillation ablation technologies in a multicenter study. J Am Coll Cardiol 58: 681-688.
7. Demolin JM, Eick OJ, Munch K, Koullick E, Nakagawa H, et al. (2002) Soft thrombus formation in radiofrequency catheter ablation. Pacing Clin Electrophysiol 25: 1219-1222.

8. Khairy P, Chauvet P, Lehmann J, Jean Lambert, Laurent Macle, et al. ((2003) Lower incidence of thrombus formation with cryoenergy versus radiofrequency catheter ablation. Circulation 107: 2045-2050.

9. Christian-Hendrik Heeger, Andreas Metzner, Michael Schlüter, Andreas Rillig, Shibu Mathew, et al. (2018) Cerebral Protection During Catheter Ablation of Ventricular Tachycardia in Patients With Ischemic Heart Disease. J Am Heart Assoc 7(13): e009005.

10. Wang YF, Cen XJ, Yu JW, Qu BM, Wang LH (2017) Aeroembolism in left atrium during catheter ablation of atrial fibrillation in a patient with dextrocardia: a case report and review of the literature. BMC Cardiovasc Disord 17(1): 143.

11. David A Igel, Jon F Urban, James P Kent, Bernard Lim, KL Venkatachalam, et al. (2018) Effect of Charge Delivery on Thromboembolism During Radiofrequency Ablation in Canines. JACC Clin Electrophysiol 4: 958966.

12. Lim B, Venkatachalam KL, Henz BD, Johnson SB, Jahangir A, et al. (2016) Prevention of Coagulum Formation With Simultaneous Charge Delivery in Radiofrequency Ablation: A Canine Model. JACC Clin Electrophysiol 2: 233-241.

13. Lim B, Venkatachalam KL, Jahangir A, Johnson SB, Asirvatham SJ (2008) Concurrent application of charge using a novel circuit prevents heat-related coagulum formation during radiofrequency ablation. J Cardiovasc Electrophysiol 19: 843-850.

14. Chance M Witt, Alan Sugrue, Deepak Padmanabhan, Vaibhav Vaidya, Sarah Gruba, et al. (2018) Intrapulmonary Vein Ablation Without Stenosis: A Novel Balloon-Based Direct Current Electroporation Approach. J Am Heart Assoc 7(14): e009575.

15. Stephan Haussig, Norman Mangner, Michael G Dwyer, Lukas Lehmkuhl, Christian Lücke, et al. (2016) Effect of a Cerebral Protection Device on Brain Lesions Following Transcatheter Aortic Valve Implantation in Patients With Severe Aortic Stenosis: The CLEAN-TAVI Randomized Clinical Trial. JAMA 316: 592-601.

16. Samir R Kapadia, Susheel Kodali, Raj Makkar, Roxana Mehran, Ronald M Lazar, et al. (2017) Protection Against Cerebral Embolism During Transcatheter Aortic Valve Replacement. J Am Coll Cardiol 69: 367-377.

17. Ndunda PM, Vindhyal MR, Muutu TM, Fanari Z (2019) Clinical outcomes of sentinel cerebral protection system use during transcatheter aortic valve replacement: A systematic review and meta-analysis. Cardiovasc Revasc Med 21(6): 717-722.

18. Schafer U (2017) Safety and Efficacy of Protected Cardiac Intervention: Clinical Evidence for Sentinel Cerebral Embolic Protection. Interv Cardiol 12: 128-132.

19. Nicolas M Van Mieghem, Lennart van Gils, Habib Ahmad, Floortje van Kesteren, Hendrik W van der Werf, et al. (2016) Filter-based cerebral embolic protection with transcatheter aortic valve implantation: the randomised MISTRAL-C trial. EuroIntervention 12: 499-507.

20. Wang N, Phan K (2018) Cerebral protection devices in transcatheter aortic valve replacement: a clinical meta-analysis of randomized controlled trials. J Thorac Dis 10: 1927-1935.

21. Deepak Padmanabhan, Alan Sugrue, Vaibhav Vaidya, Chance Witt, Omar Yasin, et al. (2020) Incremental benefit of a novel signal recording system during mapping and ablation. Europace 23(1): 130-138. 